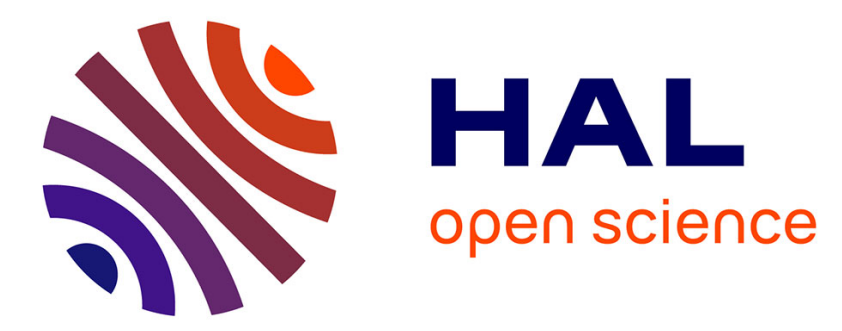

\title{
Quasi-periodic harmonic balance method for rubbing self-induced vibrations in rotor-stator dynamics
}

\author{
Loïc Peletan, Sébastien Baguet, Mohamed Torkhani, Georges
}

Jacquet-Richardet

\section{To cite this version:}

Loïc Peletan, Sébastien Baguet, Mohamed Torkhani, Georges Jacquet-Richardet. Quasi-periodic harmonic balance method for rubbing self-induced vibrations in rotor-stator dynamics. Nonlinear Dynamics, 2014, 78 (4), pp.2501-2515. 10.1007/s11071-014-1606-8 . hal-01061265

\section{HAL Id: hal-01061265 \\ https://hal.science/hal-01061265}

Submitted on 8 Sep 2015

HAL is a multi-disciplinary open access archive for the deposit and dissemination of scientific research documents, whether they are published or not. The documents may come from teaching and research institutions in France or abroad, or from public or private research centers.
L'archive ouverte pluridisciplinaire HAL, est destinée au dépôt et à la diffusion de documents scientifiques de niveau recherche, publiés ou non, émanant des établissements d'enseignement et de recherche français ou étrangers, des laboratoires publics ou privés. 


\title{
Quasi-Periodic Harmonic Balance Method for Rubbing Self-Induced Vibrations in Rotor-Stator Dynamics
}

\author{
Loïc Peletan · Sébastien Baguet • Mohamed Torkhani · Georges Jacquet-Richardet
}

Received: date / Accepted: date

\begin{abstract}
A quasi-periodic Harmonic Balance Method (HBM) coupled with a pseudo-arc length continuation algorithm is developed and used for the prediction of the steady state dynamic behaviour of rotor-stator contact problems. Quasiperiodic phenomena generally involve two incommensurable fundamental frequencies and at present the Harmonic Balance Method has been adapted to deal with cases where those frequencies are known. The problem here is to improve the procedure in order to be able to deal with cases where one of the two fundamental frequencies is a priori unknown, in order to be able to reproduce self-excited phenomena such as the so-called quasi-periodic partial rub. Considering the proposed developments, the unknown fundamental frequency is automatically determined during calculation and an automatic harmonic selection procedure gives both accuracy and performance improvements. The application is based on a Jeffcott rotor model and results obtained are compared with traditional time marching solutions. The modified quasi-periodic HBM appears one order of magnitude faster than transient simulations while providing very accurate results.
\end{abstract}

Keywords quasi-periodic harmonic balance method . self-induced excitation - automatic harmonic selection . nonlinear rotordynamics $\cdot$ rotor-stator contact

L. Peletan $\cdot$ S. Baguet $\cdot$ G. Jacquet-Richardet

Université de Lyon, CNRS, INSA-Lyon, LaMCoS UMR5259, F69621, Villeurbanne cedex, France

E-mail: loic.peletan@insa-lyon.fr

E-mail: sebastien.baguet@insa-lyon.fr

E-mail: georges.jacquet@insa-lyon.fr

M. Torkhani

LaMSID UMR EDF-CNRS-CEA 2832, EDF R\&D, F-92141 Clamart

Cedex, France

E-mail: mohamed.torkhani@edf.fr

\section{Introduction}

Improving turbomachines efficiency may be achieved by reducing the operating gap between rotating and stationary parts (rotor and stator). However this leads to an increased risk of rotor-to-stator contact and, for safety reasons, designers must ensure that such interactions cannot have serious consequences. When an accurate prediction of the system response is required, it is not sufficient to consider linear models. When nonlinearities are considered, highly complex dynamic behaviours are possible, even in the case of very simple rotors [1]. The simplest rotor model encountered is the Jeffcott model described by only one node and two degrees of translational freedom (one for each radial direction). Edwards [2] studied a Jeffcott rotor with an additional torsional degree of freedom, when subjected to a mass unbalance and contact with a circular, rigid, fixed stator. He modelled the contact using a penalty method with a Coulomb friction law and showed that even when the rotor is only excited by a periodic force, this simple system may exhibit a rich variety of phenomena due to the presence of nonlinearity.

Many authors have studied nonlinear rotor models, using time marching techniques [2-6]. This approach, although essential when investigating the system's transient behaviour $[3,7,8]$, is not optimal in terms of CPU time when the steady state response is investigated, because many cycles need to be simulated before the transient behaviour disappears completely. This can be problematic when parametric studies need to be carried out and/or when more complex models are used (such as finite element rotor models [5,9]).

On the other hand, some authors such as Jiang $[10,11]$ or Bently et al. [12] use analytical tools to study simple rotors and determine the limits of existence for different types of behaviours, according to a set of dimensionless parameters. Considering Jiang's model the stator is circular, rigid and 


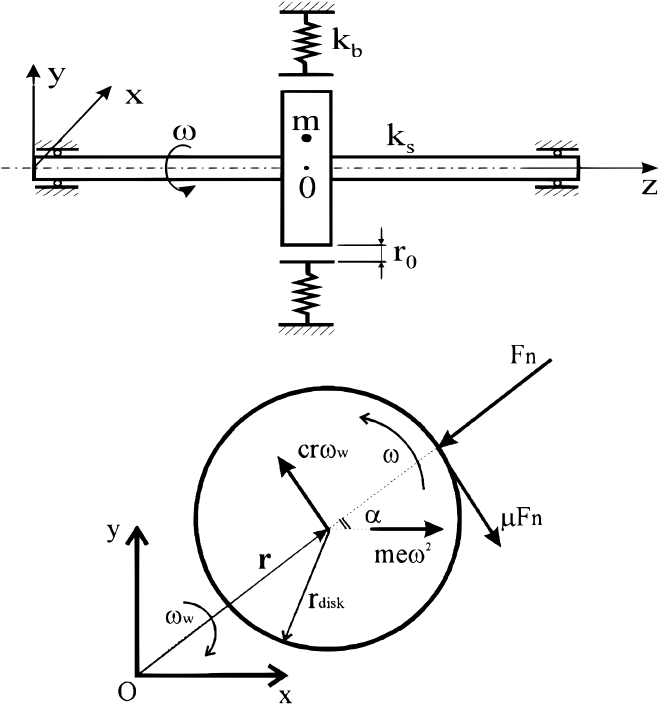

Fig. 1 Schematic representation of a Jeffcott rotor with a circular rigid stator, after [11].

fixed and contact is modelled by a penalty law using a dry friction Coulomb approach (Fig. 1). His model is able to exhibit the four classical types of steady-state rotor behaviour, resulting from unbalance and rotor-to-stator contact. These four types of behaviour are:

- 'no-rub motion' in which there is no contact between the rotor and the stator. The orbit is circular and the motion is periodic.

- 'full annular rub' in which the rotor remains in permanent contact with the stator. The orbit is also circular and the motion periodic.

- 'partial rub' in which the rotor intermittently comes into contact with the stator. The motion is quasi-periodic.

- 'backward whip' in which the rotor rolls (with slippage) on the inner surface of the stator. This motion is selfexcited and its frequency is not necessarily a direct function of rotational speed. The phenomenon is violent and a priori quasi-periodic.

The governing equations of the rotor/stator contact system can be written as

$$
\begin{aligned}
X^{\prime \prime} & +2 \xi X^{\prime}+\beta X+\Theta\left(1-\frac{R_{0}}{R}\right)\left(X-\mu \operatorname{sign}\left(V_{\text {rel }}\right) Y\right) \\
& =\Omega^{2} \cos \Omega \tau \\
Y^{\prime \prime} & +2 \xi Y^{\prime}+\beta Y+\Theta\left(1-\frac{R_{0}}{R}\right)\left(\mu \operatorname{sign}\left(V_{\text {rel }}\right) X+Y\right) \\
& =\Omega^{2} \sin \Omega \tau \\
V_{\text {rel }} & =R_{\text {disk }} \Omega+R \omega_{b}
\end{aligned}
$$

Jiang's study [11] is based on the following dimensionless variables: $X=\frac{x}{e}, Y=\frac{y}{e}, R_{0}=\frac{r_{0}}{e}, R_{\text {disk }}=\frac{r_{\text {disk }}}{e}, V_{\text {rel }}=\frac{v_{\text {rel }}}{e}$, $2 \xi=\frac{c}{\sqrt{k_{b} m}}, \beta=\frac{k_{s}}{k_{b}}, \omega_{s}=\sqrt{\frac{k_{b}}{m}}, \Omega=\frac{\omega}{\omega_{s}}, \omega_{b}=\frac{\omega_{w}}{\omega_{s}}$,

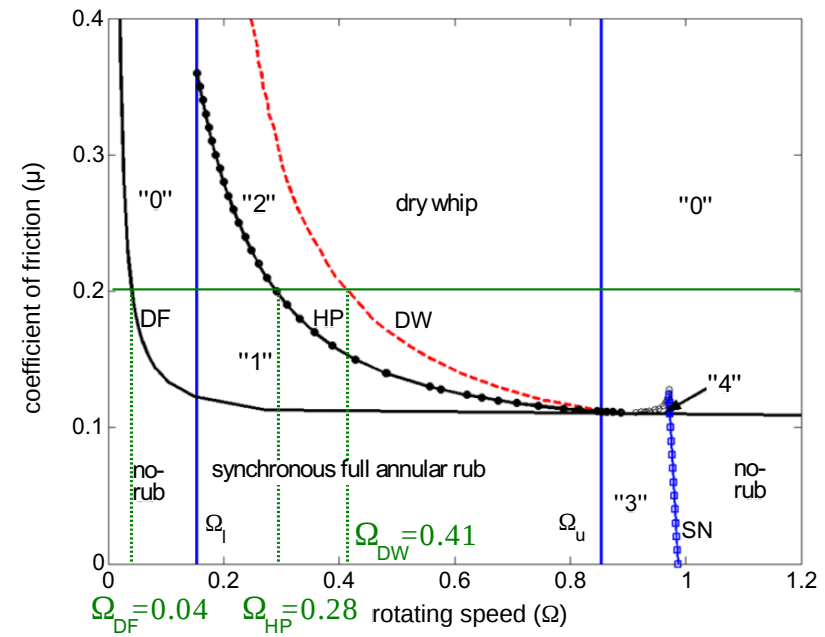

Fig. 2 Rotor response characteristics in the $\Omega-\mu$ plane with $\beta=0.04$, $\xi=0.05, R_{0}=1.05$ and $R_{\text {disk }}=20 R_{0}$, after [11]. Some characteristic values of $\Omega$ associated to $\mu=0.2$ are highlighted.

where $x$ and $y$ represent the radial displacements along the $\mathrm{x}$ and $\mathrm{y}$ axes respectively, $e$ is the mass eccentricity, $r_{0}$ the initial clearance between the rotor and the stator, $r_{\text {disk }}$ the disk radius, $v_{\text {rel }}$ the relative velocity between the rotor and the stator, $c$ the damping factor, $m$ the mass of the rotor, $k_{s}$ and $k_{b}$ are the stiffnesses of the rotor and stator respectively, $\omega$ is the rotational speed of the rotor, $\omega_{w}$ the whirl frequency of the rotor and $\omega_{s}$ the natural frequency of the rotor system without clearance. The coefficient of friction is denoted $\mu$.

Fig. 2 is extracted from Jiang's paper [11]. It shows the analytical prediction of the response characteristics of the rotor in the $\Omega$ - $\mu$ plane. The straight lines $\Omega_{l}$ and $\Omega_{u}$ indicate the rotational speed limits where rotor eccentricity reaches the initial clearance. Thus, the no-rub motion can appear only at rotational speeds below $\Omega_{l}$ and above $\Omega_{u}$. The curves $\mathrm{HP}$ and SN were obtained numerically and correspond to the limits of full annular rub motion. To the right of line HP, the full annular rub becomes unstable and motion becomes quasi-periodic (Hopf bifurcation). To the right of line SN, the full annular rub no longer exists (saddle node bifurcation). Line DF is the backward whip limit and line DW indicates the point beyond which backward whip is triggered by unbalance. Some of Jiang's analytical equations had to be solved with the help of numerical calculations. It explains the dots on the HP and SN curves.

There are some regions in which several behaviours coexist. Within regions labelled '0' no-rub and dry whip may exist. In the region labelled ' 1 ', both full annular rub and dry friction backward whirl are possible. The region labelled '2' shows the presence of partial rub and backward whirl. In '3', full annular rub and no-rub motions are simultaneously present. Finally, a small region '4' exhibits no-rub, full annular rub and backward whip possibilities. Time integration 
methods would lead to only one of these solutions, depending on initial conditions.

Analytical methods have been extended to multi-mode rotor models by Childs and Bhattacharya [13]. Their predictions appear to be in good agreement with numerical simulations and experiments. Nevertheless, these analytical methods require non-trivial mathematical developments and their applicability is limited when considering complex models.

Alternatively, although widely used to solve other types of problems, numerical techniques such as shooting or the Harmonic Balance Methods (HBM) are rarely used for rotorstator contact problems. Although such techniques do not require long transient simulations and are in practice much faster, they are usually limited to the study of periodic motions. This limitation explains that those techniques are generally restricted to the study of geometric nonlinearities or oil-film bearing nonlinearities [14], because with this kind of nonlinearities the system response remains mostly periodic. Fortunately, extension of the HBM to quasi-periodicity is possible by introducing the concept of multidimensional time and has already been performed for various applications. Lau [15] studied the quasi-periodic free response of a clamped beam with large displacements. Legrand [16,17] used the quasi-periodic HBM for the computation of limit cycles for autonomous systems with application to aircraft turbomachinery with rotor-stator interaction. Coudeyras [18] used a similar approach applied to the study of break squeal. Complex nonlinear normal modes can be computed with the help of quasi-periodic HBM as done by Laxalde and Thouverez [19] for blade-casing contact problems. The quasiperiodic HBM coupled with an arc-length continuation scheme was also used by Guskov [20] for the forced response of a multiple shaft rotor subject to quasi-periodic multi-excitations with known frequencies. Some variants can be found in the literature. For example, instead of computing a quasi-periodic trajectory which takes place on the surface of an invariant torus, Schilder et al. [21] focus on the direct computation of the torus itself and solve a so-called Invariance PDE that a torus function must satisfy, with application to non-linear electrical engineering.

In the following, the nonlinear Jeffcott rotor model described in [11] is first considered and analysed using a classical time marching procedure. The goal is to simulate the transient rotor behaviour in order to analyse carefully the responses obtained. The highlighted important results lead to the proposition of an improved Harmonic Balance Method associated with a pseudo arc-length continuation technique, able to reproduce efficiently quasi-periodic phenomena where nothing is a priori known concerning the frequency induced by the contact mechanism.

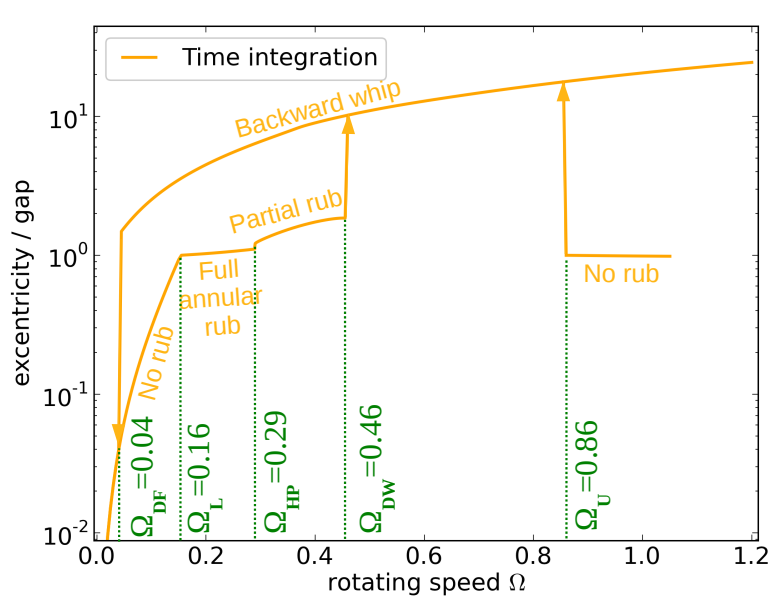

Fig. 3 Response curve for $\mu=0.2$.

\section{Nonlinear Jeffcott rotor - numerical approach}

The results shown in Fig. 3 have been examined via numerical simulations based on a time marching procedure. Considering the same parameter values as those presented in Fig. 2, simulations were run with a fixed value for the friction coefficient $\mu$ and varying values of $\Omega$. Solutions were calculated for discrete values of $\Omega$ and the initial conditions used at each step were given by the final steady state solution of the previous one (incremental continuation technique). For each value of $\Omega, 100$ revolutions were simulated in order to reach the steady-state behaviour. Twenty-five additional revolutions were then simulated and saved for analysis. This procedure was performed twice for each value of $\mu$. In the first round, simulations were made with progressively greater values of $\Omega$, whereas in the second progressively smaller values of $\Omega$ were used. As numerical simulations require the use of non dimensionless variables, the following parameters were set arbitrarily: $e=0.1 \mathrm{~m}, k_{s}=100$ $\mathrm{N} . \mathrm{m}^{-1}, m=1 \mathrm{~kg}$ and the other parameters deduced from those. All the simulations were performed using Code_Aster [22] and user scripts in Scipy [23] environments. Although simulations involved many values of $\mu$, only the results obtained for $\mu=0.2$, which are representative of all possible solutions, are presented in the following. The response curve obtained for $\mu=0.2, \beta=0.04, \xi=0.05, R_{0}=1.05$ and $R_{\text {disk }}=20 R_{0}$ is shown in Fig. 3. The y-axis represents rotor eccentricity normalized with the initial clearance, on a logarithmic scale, and the $\mathrm{x}$-axis is the normalized rotational speed $\Omega=\frac{\omega}{\omega_{s}}$. Different behaviours are obtained according to the analytical prediction (Fig. 2).

During the sweep-up, the no-rub motion is first observed (Fig. 4). At $\Omega_{l} \approx 0.16$, the full annular rub regime starts with a rotor eccentricity slightly larger than the initial gap due to the contact elasticity (Fig. 5). The transition between 


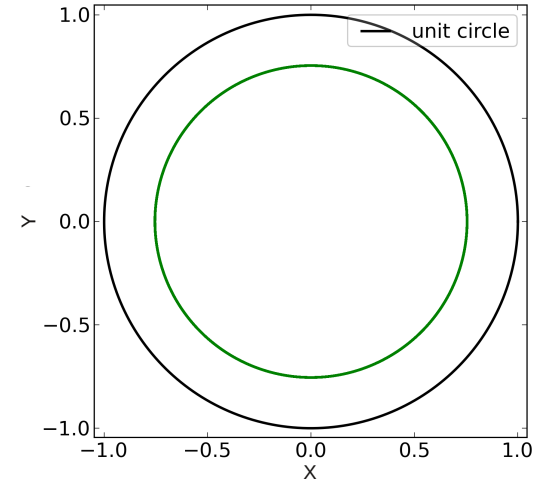

Fig. 4 Orbit during the no rub motion at $\Omega=0.14$

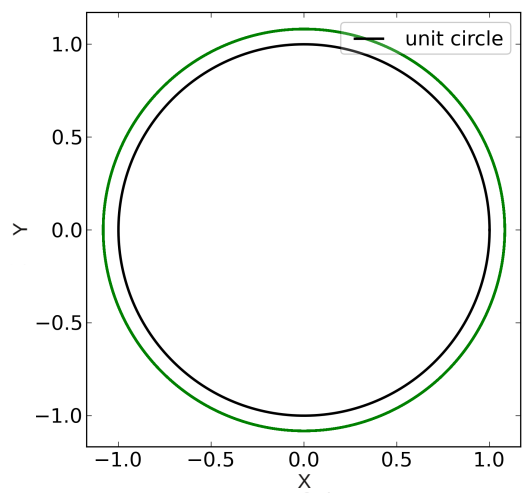

Fig. 5 Orbit during full annular rub at $\Omega=0.26$

no-rub and full annular rub (which corresponds to the $\Omega_{l}$ line in Fig. 2) occurs between approximately $\Omega=0.155$ and $\Omega=0.160$, which is highly consistent with the analytical value ( $\Omega_{l}=0.1536$ ). Beyond $\Omega_{\mathrm{HP}} \approx 0.29$, rotor motion becomes quasi-periodic. The orbit at $\Omega=0.33$ is shown in Fig. 6 and illustrates the partial rub motion predicted, where the rotor does not remain in permanent contact with the stator and the maximum rotor eccentricity is greater than that predicted during full annular rub. At $\Omega_{\mathrm{DW}} \approx 0.46$, eccentricity suddenly increases by a factor of ten. Although slightly different from the value found analytically $(\approx 0.41)$, the value obtained is coherent. In this backward dry whip regime, rotor's orbit is almost circular, as shown in Fig. 7. The rotor then remains in the backward whip regime until the end of the sweep-up.

During the sweep-down, the rotor starts with the no-rub regime, until $\Omega_{u} \approx 0.86$ where a sudden increase in eccentricity occurs, transferring the motion of the rotor to the backward whip regime. The rotor remains in this regime until a very small value of $\Omega_{\mathrm{DF}} \approx 0.04$ is reached, at which eccentricity suddenly drops leading to the no rub regime. This value of $\Omega_{\mathrm{DF}}$ is also in good agreement with the analytical value (Fig. 2). Transient simulations are used to analyse more deeply the nature of the quasi-periodic regimes. A Fast Fourier Transform of the nonlinear contact force, during partial rub at $\Omega=0.33$, is shown Fig. 8 . When analysing

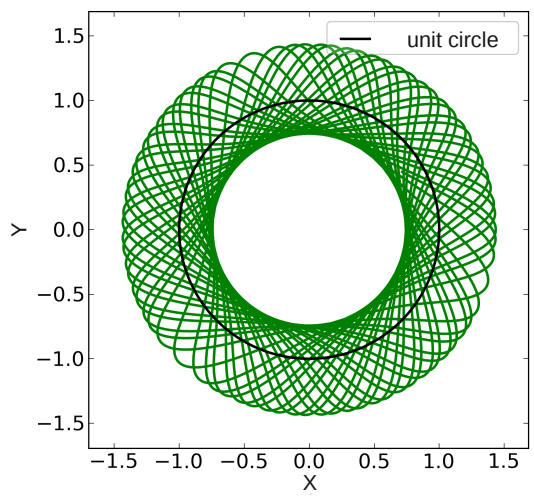

Fig. 6 Orbit during partial rub at $\Omega=0.33$

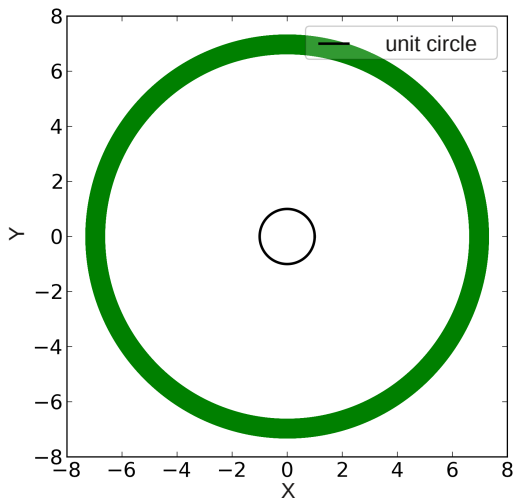

Fig. 7 Backward whip orbit at $\Omega=0.33$. Rotor's eccentricity is one order of magnitude greater than clearance.

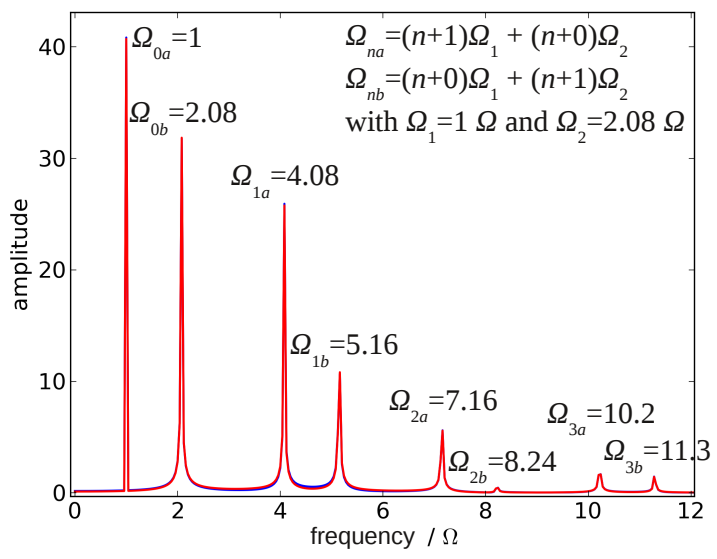

Fig. 8 Fast Fourier transform of the nonlinear contact force signal during partial rub at $\Omega=0.33$.

those results, it appears that the frequencies obtained are all linked by a linear combination of two incommensurable frequencies. The lowest frequency $\Omega_{1}$ is associated to rotation $(1 \times \Omega)$ while the second $\Omega_{2}$ cannot be determined a priori since it is not commensurable with $\Omega_{1}$, here $\Omega_{2} \approx 2.08 \times \Omega$. For every simulated partial rub motions, spectral peaks appear in the contact force power spectrum at linear combinations of the two incommensurable frequencies, according to 


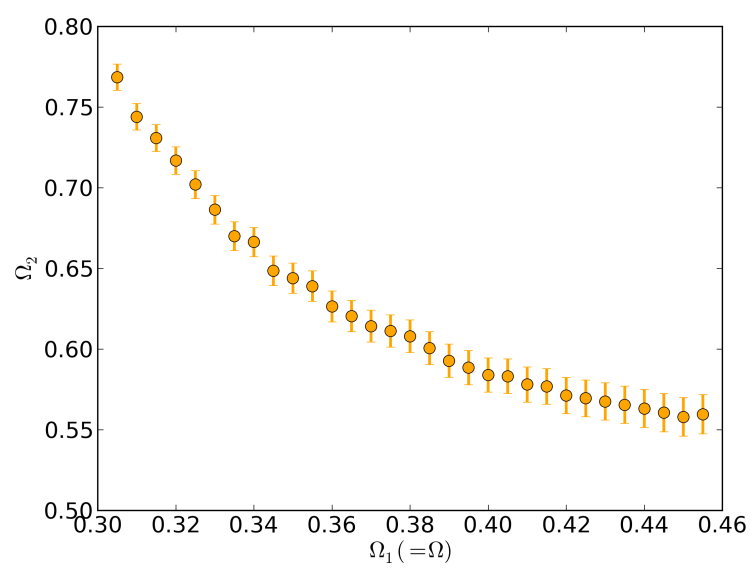

Fig. 9 Evolution of the second fundamental frequency $\Omega_{2}$ as a function of $\Omega_{1}$ with error bars.

Table $1 \Omega_{n a}$ and $\Omega_{n b}$ calculated for the first four values of $n$ for partial rub motion at $\Omega=0.33$. Here, $\Omega_{1}=1 \times \Omega$ and $\Omega_{2} \approx 2.08 \times \Omega$.

\begin{tabular}{ccc}
\hline$n$ & $\Omega_{n a}(\times \Omega)$ & $\Omega_{n b}(\times \Omega)$ \\
\hline 0 & 1 & 2.08 \\
1 & 4.08 & 5.16 \\
2 & 7.16 & 8.24 \\
3 & 10.2 & 11.3 \\
\hline
\end{tabular}

the following relations:

$\Omega_{n a}=(n+1) \Omega_{1}+(n+0) \Omega_{2} \quad$ for $n \in \mathbb{N}$

$\Omega_{n b}=(n+0) \Omega_{1}+(n+1) \Omega_{2}$ for $n \in \mathbb{N}$

Peaks appear in pairs and decrease in amplitude with $n$ (Fig. 8). Values of $\Omega_{n a}$ and $\Omega_{n b}$ are given in Table 1 and are coherent with those highlighted in Fig. 8.

Spectral analysis of the contact force during partial rub for several values of $\Omega$ shows not only that $\Omega_{1}$ remains equal to the rotating frequency $\left(\Omega_{1}=1 \times \Omega\right)$, but also that $\Omega_{2}$ is related to $\Omega_{1}$ without any trivial relationship $\left(\Omega_{2}=f\left(\Omega_{1}\right)\right.$ ). The only evident point is that $\Omega_{2}$ decreases when $\Omega$ increases. The evolution of $\Omega_{2}$ with $\Omega_{1}$ is given in Fig. 9. A nonlinear decrease of $\Omega_{2}$ with $\Omega_{1}$ is observed. As the two fundamental frequencies are incommensurable and due to limited spectral resolution, only approximate measurements of $\Omega_{2}$ can be obtained, with lower and upper bounds indicated by error bars in Fig. 9.

Those direct simulations are highly computer time demanding. On the other hand the harmonic balance method is very efficient but is limited to the study of periodic solutions or quasi-periodic solutions in the case where the relationship between the different frequency components is known. In the following, after a brief presentation of the periodic HBM and the pseudo arc-length continuation method, an extension of the HBM is proposed. As required here, the method handles situations where one of the two fundamental frequencies is a priori unknown.

\section{Periodic Harmonic Balance Method (HBM)}

\subsection{Basics of the HBM}

The classical Harmonic Balance Method (HBM) is used to compute periodic solutions of dynamical systems. With this method the equations of motion are solved in the frequency domain, rather than in the time domain. The general equation for dynamical systems is:

$\mathbf{M} \ddot{\mathbf{q}}(t)+\mathbf{C} \dot{\mathbf{q}}(t)+\mathbf{K q}(t)+\mathbf{f}(\mathbf{q}(t), \dot{\mathbf{q}}(t))-\mathbf{p}(t)=\mathbf{0}$

where $\mathbf{q}$ is the displacement vector for all of the $n$ degrees of freedom; K, $\mathbf{C}$ and $\mathbf{M}$ are the generalized $n \times n$ stiffness, damping and mass matrices; $\mathbf{f}$ is the nonlinear force vector and $\mathbf{p}$ the external excitation force vector.

When the external excitations are periodic, it is possible to assume that a steady state solution for Eq. (4) exists, and that this solution is also periodic. The displacements, the external and the nonlinear forces can thus be written as truncated Fourier series with $N$ harmonics:

$\mathbf{q}(t)=\mathbf{Q}_{0}+\sum_{j=1}^{N}\left(\mathbf{Q}_{2 j-1} \cos (j \omega t)+\mathbf{Q}_{2 j} \sin (j \omega t)\right)$

Not all the harmonics contribute to the solution in the same way. Some harmonics are prevailing while some others have a nil contribution. If only the harmonics of interest (see section 4.3 for harmonic selection) are retained in the solution, then the truncated Fourier series for displacements, external and nonlinear forces become:

$$
\begin{aligned}
& \mathbf{q}(t)=\mathbf{Q}_{0}+\sum_{j=1}^{N}\left(\mathbf{Q}_{2 j-1} \cos \left(m_{j} \omega t\right)+\mathbf{Q}_{2 j} \sin \left(m_{j} \omega t\right)\right) \\
& \mathbf{p}(t)=\mathbf{P}_{0}+\sum_{j=1}^{N}\left(\mathbf{P}_{2 j-1} \cos \left(m_{j} \omega t\right)+\mathbf{P}_{2 j} \sin \left(m_{j} \omega t\right)\right) \\
& \mathbf{f}(t)=\mathbf{F}_{0}+\sum_{j=1}^{N}\left(\mathbf{F}_{2 j-1} \cos \left(m_{j} \omega t\right)+\mathbf{F}_{2 j} \sin \left(m_{j} \omega t\right)\right)
\end{aligned}
$$

$\mathbf{Q}=\left[\mathbf{Q}_{\mathbf{0}}{ }^{T}, \mathbf{Q}_{\mathbf{1}}{ }^{T}, \ldots, \mathbf{Q}_{2 N}^{T}\right]^{T}, \mathbf{F}=\left[\mathbf{F}_{0}^{T}, \mathbf{F}_{1}^{T}, \ldots, \mathbf{F}_{2 N}^{T}\right]^{T}$ and $\mathbf{P}=\left[\mathbf{P}_{0}^{T}, \mathbf{P}_{1}^{T}, \ldots, \mathbf{P}_{2 N}^{T}\right]^{T}$ are vectors of the Fourier coefficients for displacements, nonlinear forces and external excitations, respectively. $\omega$ is the fundamental frequency of the external excitation, and $m_{j} \in \mathbb{N}(1 \leq j \leq N)$ represent the indexes of the $N$ harmonics of interest retained in the solution.

As described in [24] for example, Eqs. (5) (6) and (7) can be substituted into Eq. (4) and a Galerkin procedure applied to transform the nonlinear differential Eq. (4) of dimension $n$, into an algebraic nonlinear system of dimension $n_{\mathrm{HBM}}=$ $n(2 N+1)$ :

$\mathbf{R}(\mathbf{Q}, \omega)=\mathbf{Z}(\omega) \mathbf{Q}+\mathbf{F}(\mathbf{Q})-\mathbf{P}=\mathbf{0}$ 
where $\mathbf{Z}(\omega)=\operatorname{diag}\left(\mathbf{K}, \mathbf{Z}_{1}(\omega), \ldots, \mathbf{Z}_{j}(\omega), \ldots, \mathbf{Z}_{N}(\omega)\right)$ and: $\mathbf{Z}_{j}(\omega)=\left[\begin{array}{cc}\mathbf{K}-\left(m_{j}\right)^{2} \omega^{2} \mathbf{M} & \left(m_{j}\right) \omega \mathbf{C} \\ -\left(m_{j}\right) \omega \mathbf{C} & \mathbf{K}-\left(m_{j}\right)^{2} \omega^{2} \mathbf{M}\end{array}\right]$

Eq. (8) has to be solved for $\mathbf{Q}$. As this equation is still nonlinear, an incremental-iterative Newton-Raphson method is used to derive a correct solution.

The Alternating Frequency Time (AFT) algorithm [25] gives an efficient way to compute the nonlinear term $\mathbf{F}(\mathbf{Q})$ and its derivative $\partial \mathbf{F}(\mathbf{Q}) / \partial \mathbf{Q}$ at each Newton-Raphson iteration. The nonlinear forces are usually much easily evaluated in the time domain than in the frequency domain. Moreover, the AFT scheme uses fast direct and inverse Fourier transforms to quickly compute the nonlinear forces in the time domain and then switch back to the frequency domain. A combination with a pseudo-arc length continuation [14] allows following efficiently the solution branches. The stability of the solutions is determined using the Floquet's theory [26].

\subsection{Pseudo arc-length continuation method}

The interest of the pseudo arc-length continuation method lies in its ability to follow response branches beyond turning points and thus completely describe a complex solution branch with potentially several solutions for a given value of $\omega$. Let $M_{(i)}\left(\mathbf{Q}_{(i)}, \omega_{(i)}\right)$ be a point that satisfies $\mathbf{R}\left(\mathbf{Q}_{(i)}, \omega_{(i)}\right)=$ 0. Then the goal is to obtain a point $M_{(i+1)}\left(\mathbf{Q}_{(i+1)}, \omega_{(i+1)}\right)$ that also satisfies $\mathbf{R}\left(\mathbf{Q}_{(i+1)}, \omega_{(i+1)}\right)=\mathbf{0}$, with $\mathbf{Q}_{(i+1)}=\mathbf{Q}_{(i)}+$ $\Delta \mathbf{Q}_{(i)}$ and $\omega_{(i+1)}=\omega_{(i)}+\Delta \omega_{(i)}$. The continuation procedure consists in two steps: a prediction and corrections.

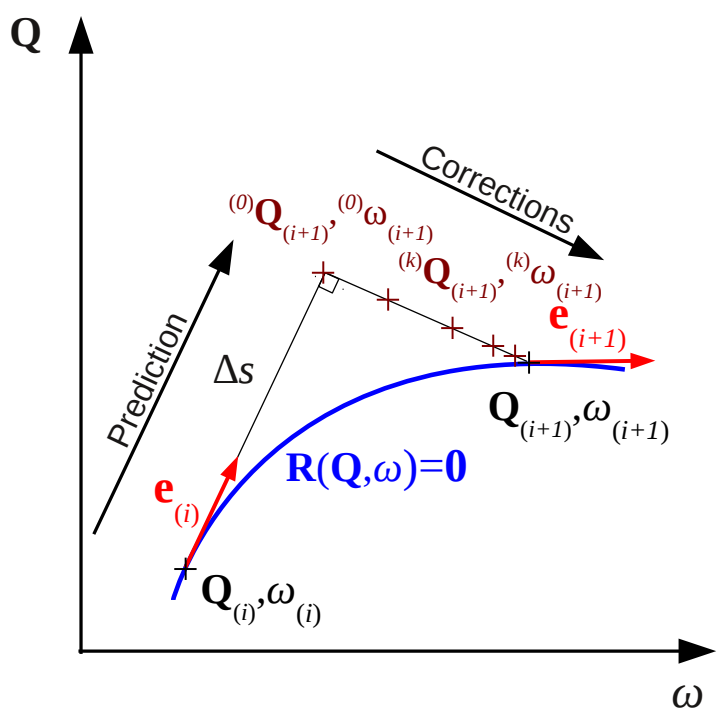

Fig. 10 Pseudo arc-length continuation.

\subsubsection{Prediction}

During the prediction step, the solution for the next value of $\omega$ is assessed. The prediction is made in a direction tangent to the solution branch. The following first order approximation can be made:

$$
\begin{aligned}
& \underbrace{\mathbf{R}\left(\mathbf{Q}_{(i+1)}, \omega_{(i+1)}\right)}_{=0} \approx \\
& \underbrace{\mathbf{R}\left(\mathbf{Q}_{(i)}, \omega_{(i)}\right)}_{=0}+\left.\Delta \mathbf{Q}_{(i)} \frac{\partial \mathbf{R}}{\partial \mathbf{Q}}\right|_{M_{(i)}}+\left.\Delta \omega_{(i)} \frac{\partial \mathbf{R}}{\partial \omega}\right|_{M_{(i)}}
\end{aligned}
$$

with $\frac{\partial \mathbf{R}}{\partial \mathbf{Q}}=\mathbf{Z}(\omega)+\frac{d \mathbf{F}}{d \mathbf{Q}}$ and $\frac{\partial \mathbf{R}}{\partial \omega}=\frac{d \mathbf{Z}}{d \omega} \mathbf{Q}$.

The norm of the tangent vector $\mathbf{e}_{(i)}=\left[\Delta \mathbf{Q}_{(i)}^{T}, \Delta \omega_{(i)}\right]$ is:

$\left\|\mathbf{e}_{(i)}\right\|^{2}=\Delta \mathbf{Q}_{(i)}^{T} \Delta \mathbf{Q}_{(i)}+\Delta \omega_{(i)}^{2}$

Let consider $a_{(i)}=\Delta \omega_{(i)}, \Delta \mathbf{Q}_{(i)}=a_{(i)} \Delta \hat{\mathbf{Q}}_{(i)}$ and the norm of the tangent vector set at unity, then:

$a_{(i)}= \pm \frac{1}{\sqrt{\Delta \hat{\mathbf{Q}}_{(i)}^{T} \Delta \hat{\mathbf{Q}}_{(i)}+1}}$

The sign of $a_{(i)}$ is chosen so that two consecutive tangent vectors have a positive scalar product (direction conservation). Eq. (9) becomes:

$\left.\Delta \mathbf{Q}_{(i)} \frac{\partial \mathbf{R}}{\partial \mathbf{Q}}\right|_{M_{(i)}}+\left.\Delta \omega_{(i)} \frac{\partial \mathbf{R}}{\partial \omega}\right|_{M_{(i)}}=\mathbf{0}$

Considering the expressions of $a_{(i)}\left(a_{(i)}=\Delta \omega_{(i)}\right)$ and $\Delta \mathbf{Q}_{(i)}$ $\left(\Delta \mathbf{Q}_{(i)}=a_{(i)} \Delta \hat{\mathbf{Q}}_{(i)}\right)$, Eq. (11) becomes:

$\left.a_{(i)} \Delta \hat{\mathbf{Q}}_{(i)} \frac{\partial \mathbf{R}}{\partial \mathbf{Q}}\right|_{M_{(i)}}+\left.a_{(i)} \frac{\partial \mathbf{R}}{\partial \omega}\right|_{M_{(i)}}=\mathbf{0}$

Hence, the procedure to compute $\mathbf{e}_{(i)}$ can be summarized as follows:

- Solve $\left.\frac{\partial \mathbf{R}}{\partial \mathbf{Q}}\right|_{M_{(i)}} \Delta \hat{\mathbf{Q}}_{(i)}=-\left.\frac{\partial \mathbf{R}}{\partial \omega}\right|_{M_{(i)}}$ for $\Delta \hat{\mathbf{Q}}_{(i)}$

- Compute $a_{(i)}$ from Eq. (10)

- Deduce $\mathbf{e}_{(i)}=\left[\Delta \mathbf{Q}_{(i)}^{T}, a_{(i)}\right]$

Once the normalised tangent vector is calculated, prediction can be made:

$\mathbf{Q}_{(i+1)}^{(0)}=\mathbf{Q}_{(i)}+\Delta s_{(i)} \cdot \mathbf{e}_{(i)}$

with $\Delta s_{(i)}$ being the step size. 


\subsubsection{Corrections}

The prediction does not a priori satisfies equilibrium. It has to be corrected iteratively in order to cancel the error. The $\mathrm{k}^{\text {th }}$ iteration gives:

$\underbrace{\mathbf{R}\left(\mathbf{Q}_{(i+1)}^{(k)}, \omega_{(i+1)}^{(k)}\right)}_{=\mathbf{R}^{(k)}}=\underbrace{\mathbf{Z}\left(\omega_{(i+1)}^{(k)}\right)}_{=\mathbf{Z}^{(k)}} \mathbf{Q}_{(i+1)}^{(k)}+\underbrace{\mathbf{F}\left(\mathbf{Q}_{(i+1)}^{(k)}\right)}_{=\mathbf{F}^{(k)}}-\mathbf{P}$

The $(i+1)$ notation will now be dropped for clarity. Corrections are forced to be orthogonal to the tangent vector $\mathbf{e}$. Thus, the following system has to be solved:

$$
\left[\begin{array}{cc}
\frac{\partial \mathbf{R}^{(k)}}{\partial \mathbf{Q}} & \frac{\partial \mathbf{R}^{(k)}}{\partial \omega} \\
\Delta \hat{\mathbf{Q}}_{(i)}^{T} & \Delta \omega_{(i)}
\end{array}\right]\left[\begin{array}{c}
\Delta \mathbf{Q}^{(k+1)} \\
\Delta \omega^{(k+1)}
\end{array}\right]=\left[\begin{array}{c}
-\mathbf{R}^{(k)} \\
0
\end{array}\right]
$$

Both $\omega$ and $\mathbf{Q}$ are corrected at each iteration. Iterations are carried out until convergence. This continuation procedure is illustrated in Fig. 10.

\subsection{Application to the Jeffcott rotor}

The results provided by the HBM with pseudo arc-length continuation and stability analysis have been added to the response curve in Fig. 11. As expected, only no-rub and full annular rub motions are obtained by the periodic HBM procedure. The stability analysis shows a loss of stability of the full annular rub beyond $\Omega_{\mathrm{HP}} \approx 0.29$. The Floquet multipliers of the unstable solution at $\Omega \approx 0.3$ can be seen in Fig. 12 . A pair of Floquet multipliers are not within the unit circle, with a non-zero imaginary component indicating a Hopf bifurcation and a loss of stability. This Hopf bifurcation corresponds to the appearance of a second frequency in the real response of the rotor, and the initiation of a quasi-periodic regime. This means that although, theoretically, the periodic full annular rub response still exists beyond this point, it is in practice no longer possible to achieve this response, due to its instability. The value of $\Omega_{\mathrm{HP}}$ obtained with the HBM is very consistent with the predicted value (see Fig. 2).

The HBM is able to predict only a portion of the time transient simulations. However, stability analysis can detect unstable solutions and then the DF curve can be determined in addition to the $\Omega_{l}$ and $\Omega_{u}$ lines. The DW and DF lines cannot be predicted by the periodic HBM. Nonetheless, a perfect match is found between stable HBM solutions and time integration results. In the following it will be shown that the HBM can be extended to quasi-periodic analysis allowing the partial rub and backward whip regimes to be predicted too.

\section{Quasi-periodic HBM}

As shown above, only the external excitation frequency $\omega_{1}$ is known here and the second fundamental frequency $\omega_{2}$ remains an additionnal unknown.

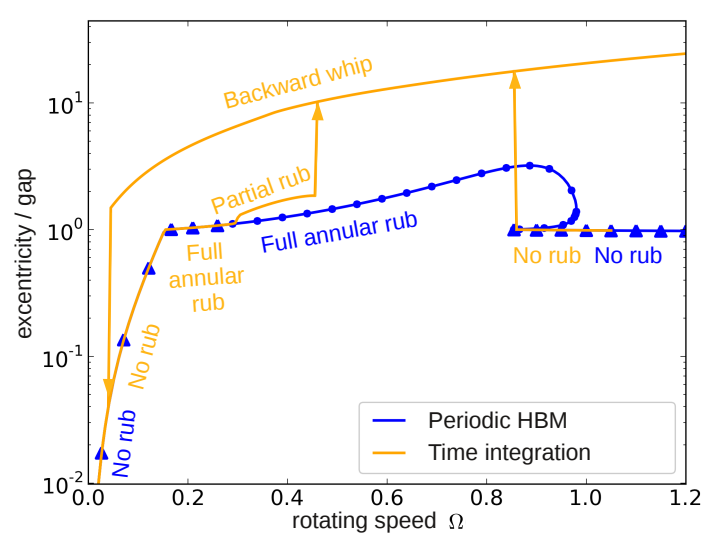

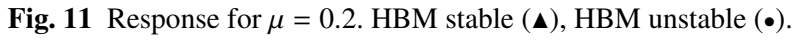

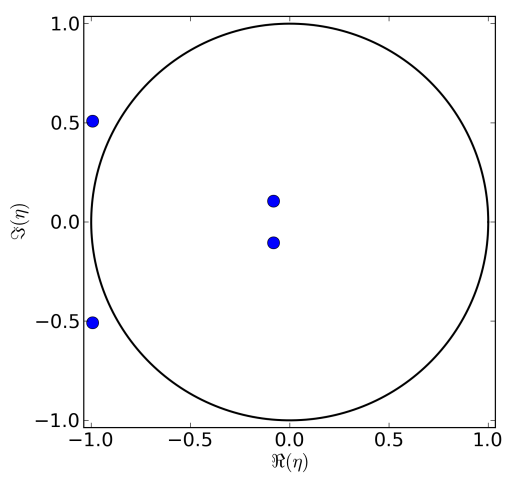

Fig. 12 Real and imaginary parts of Floquet's multipliers for the unstable solution at $\Omega \approx 0.33$ (secondary Hopf bifurcation).

\subsection{Extension of the HBM to bi-periodic solutions}

The concept of hyper-time is used to extend the Harmonic Balance Method to quasi-periodicity. When two fundamental frequencies are taken into account, the hyper-time is bidimensional and the solution is supposed to be periodic for each of the dimensions. Thus, the form of the truncated Fourier series presented in Eqs. (5), (6) and (7) must be adapted. Displacement $\mathbf{q}$ is approximated by trigonometric polynomials with two basic frequencies $\omega_{1}$ and $\omega_{2}$ :

$$
\begin{aligned}
& \mathbf{q}(t)=\mathbf{Q}_{0}+\sum_{j=1}^{N}\left(\mathbf{Q}_{2 j-1}\right. \cos \left(\left(m_{j 1} \omega_{1}+m_{j 2} \omega_{2}\right) t\right) \\
&\left.+\mathbf{Q}_{2 j} \sin \left(\left(m_{j 1} \omega_{1}+m_{j 2} \omega_{2}\right) t\right)\right)
\end{aligned}
$$

where $m_{j 1}$ and $m_{j 2} \in \mathbb{Z}(1 \leq j \leq N)$ represent the indexes of the $N$ harmonics of interest retained in the solution for $\omega_{1}$ and $\omega_{2}$ respectively. Introducing the bi-dimensional vector of basic frequencies $\omega=\left[\omega_{1}, \omega_{2}\right]$, the bi-dimensional harmonic index $\mathbf{m}_{j}=\left[m_{j 1}, m_{j 2}\right]$, and the hyper-time $\boldsymbol{\theta}=$ $\left[\theta_{1}, \theta_{2}\right]=\boldsymbol{\omega} t$, this equation can be rewritten in a form very 


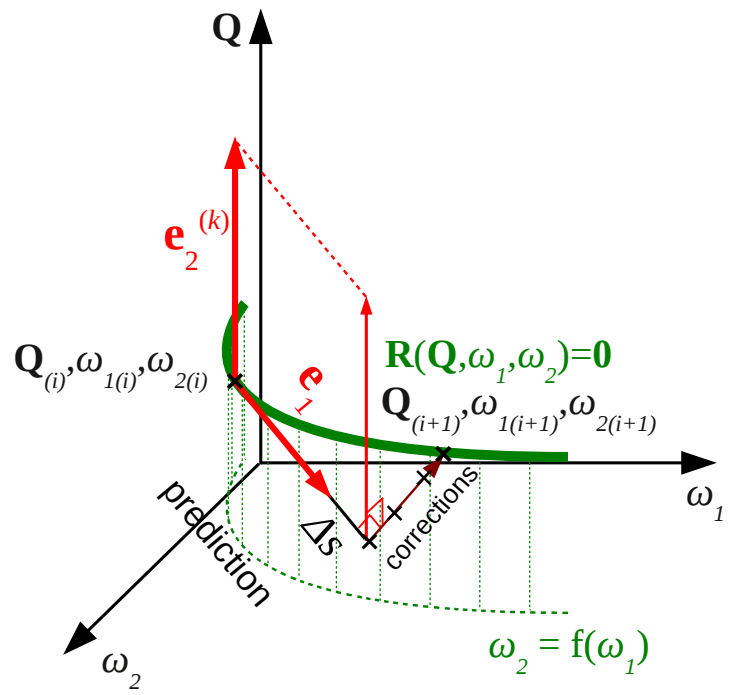

Fig. 13 Pseudo arc-length continuation with two parameters linked by an unknown function.

similar to Eq. (5) used for periodic HBM:

$\mathbf{q}(\boldsymbol{\theta})=\mathbf{Q}_{0}+\sum_{j=1}^{N}\left(\mathbf{Q}_{2 j-1} \cos \left\langle\mathbf{m}_{j}, \boldsymbol{\theta}\right\rangle+\mathbf{Q}_{2 j} \sin \left\langle\mathbf{m}_{j}, \boldsymbol{\theta}\right\rangle\right)$

where $\langle\cdot, \cdot\rangle$ stands for the classical dot product. The Galerkin procedure in this case leads to the following algebraic system:

$\mathbf{R}\left(\mathbf{Q}, \omega_{1}, \omega_{2}\right)=\mathbf{Z}\left(\omega_{1}, \omega_{2}\right) \mathbf{Q}+\mathbf{F}(\mathbf{Q})-\mathbf{P}=\mathbf{0}$

where $\mathbf{Z}\left(\omega_{\mathbf{1}}, \omega_{\mathbf{2}}\right)=\operatorname{diag}\left(\mathbf{K}, \mathbf{Z}_{1}, \ldots, \mathbf{Z}_{j}, \ldots, \mathbf{Z}_{N}\right)$ and:

$\mathbf{Z}_{j}=\left[\begin{array}{cc}\mathbf{K}-\left\langle\mathbf{m}_{j}, \boldsymbol{\theta}\right\rangle^{2} \mathbf{M} & \left\langle\mathbf{m}_{j}, \boldsymbol{\theta}\right\rangle \mathbf{C} \\ -\left\langle\mathbf{m}_{j}, \boldsymbol{\theta}\right\rangle \mathbf{C} & \mathbf{K}-\left\langle\mathbf{m}_{j}, \boldsymbol{\theta}\right\rangle^{2} \mathbf{M}\end{array}\right]$

As for periodic HBM, the purpose is to minimise the residual R. The same tools (Newton-Raphson solver, AFT, pseudo arc-length continuation) are used but need to be adapted to the quasi-periodic case. In particular, the pseudo arc-length continuation scheme must be adapted so that the second fundamental frequency is solved during the Newton-Raphson procedure.

\subsection{Extension of the pseudo arc-length continuation procedure}

The pseudo arc-length continuation algorithm is extended to a parametric study with two parameters $\left(\omega_{1}\right.$ and $\left.\omega_{2}\right)$. These two parameters are not independant but are linked by an a priori unknown function (see Fig. 13).

As before $\mathbf{M}_{(i)}\left(\mathbf{Q}_{(i)}, \omega_{1(i)}, \omega_{2(i)}\right)$ is a point that satisfies $\mathbf{R}\left(\mathbf{Q}_{(i)}, \omega_{1(i)}, \omega_{2(i)}\right)=\mathbf{0}$, and we are looking for a point $\mathbf{M}_{(i+1)}$ $\left(\mathbf{Q}_{(i+1)}=\mathbf{Q}_{(i)}+\Delta \mathbf{Q}_{(i)}, \omega_{1(i+1)}=\omega_{1(i)}+\Delta \omega_{1(i)}, \omega_{2(i+1)}=\omega_{2(i)}+\right.$ $\left.\Delta \omega_{2(i)}\right)$ that also satifies $\mathbf{R}\left(\mathbf{Q}_{(i+1)}, \omega_{1(i+1)}, \omega_{2(i+1)}\right)=\mathbf{0}$.

\subsubsection{Prediction}

Here also, the following first order approximation can be made:

$$
\begin{aligned}
& \underbrace{\mathbf{R}\left(\mathbf{Q}_{(i+1)}, \omega_{1(i+1)}, \omega_{2(i+1)}\right)}_{=0} \approx \underbrace{\mathbf{R}\left(\mathbf{Q}_{(i)}, \omega_{1(i)}, \omega_{2(i)}\right)}_{=0} \\
& \quad+\left.\Delta \mathbf{Q}_{(i)} \frac{\partial \mathbf{R}}{\partial \mathbf{Q}}\right|_{M_{(i)}}+\left.\Delta \omega_{1(i)} \frac{\partial \mathbf{R}}{\partial \omega_{1}}\right|_{M_{(i)}}+\left.\Delta \omega_{2(i)} \frac{\partial \mathbf{R}}{\partial \omega_{2}}\right|_{M_{(i)}}
\end{aligned}
$$

with,

$\frac{\partial \mathbf{R}}{\partial \mathbf{Q}}=\mathbf{Z}\left(\omega_{1}, \omega_{2}\right)+\frac{\mathbf{d} \mathbf{F}}{\mathbf{d} \mathbf{Q}}$

$\frac{\partial \mathbf{R}}{\partial \omega_{1}}=\frac{\partial \mathbf{Z}}{\partial \omega_{1}} \mathbf{Q}$

$\frac{\partial \mathbf{R}}{\partial \omega_{2}}=\frac{\partial \mathbf{Z}}{\partial \omega_{2}} \mathbf{Q}$

The norm of the tangent vector $\mathbf{e}_{1(i)}=\left[\Delta \mathbf{Q}_{(i)}^{T}, \Delta \omega_{1(i)}, \Delta \omega_{2(i)}\right]$ is:

$\left\|\mathbf{e}_{1(i)}\right\|^{2}=\Delta \mathbf{Q}_{(i)}^{T} \Delta \mathbf{Q}_{(i)}+\Delta \omega_{1(i)}^{2}+\Delta \omega_{2(i)}^{2}$

Let's denote $c_{(i)}$ the local relationship between variations of $\omega_{1}$ and $\omega_{2}$ so that $c_{(i)}=\frac{\Delta \omega_{2}}{\Delta \omega_{1}}$. As this relationship is unknown, the following finite differences approximation can be made, using the previouly calculated points:

$c_{(i)}=\frac{\omega_{2(i)}-\omega_{2(i-1)}}{\omega_{1(i)}-\omega_{1(i-1)}}$

The developments are totally similar to those previously presented with $a_{(i)}=\Delta \omega_{1(i)} \sqrt{1+c_{(i)}^{2}}$ and $\Delta \mathbf{Q}_{(i)}=a_{(i)} \Delta \hat{\mathbf{Q}}_{(i)}$. In this case, the procedure can be summarised as follows:

- Solve the following system for $\Delta \hat{\mathbf{Q}}_{(i)}$ :

$$
\left.\frac{\partial \mathbf{R}}{\partial \mathbf{Q}}\right|_{M_{(i)}} \Delta \hat{\mathbf{Q}}_{(i)}=-\frac{1}{\sqrt{1+c_{(i)}^{2}}}\left(\left.\frac{\partial \mathbf{R}}{\partial \omega_{1}}\right|_{M_{(i)}}+\left.c_{(i)} \frac{\partial \mathbf{R}}{\partial \omega_{2}}\right|_{M_{(i)}}\right)
$$

- Compute $a_{(i)}$ from Eq. (10)

- Deduce $\Delta \omega_{1(i)}$ and $\Delta \omega_{2(i)}$ :

$$
\Delta \omega_{1(i)}=\frac{a_{(i)}}{\sqrt{1+c_{(i)}^{2}}} \quad ; \quad \Delta \omega_{2(i)}=c_{(i)} \Delta \omega_{1(i)}
$$

This gives vector $\mathbf{e}_{1}=\left[\Delta \hat{\mathbf{Q}}_{(i)}, \Delta \omega_{1(i)}, \Delta \omega_{2(i)}\right]$. The prediction of point $\mathbf{M}_{(i+1)}$ is made at a distance $\Delta s$ from $\mathbf{M}_{i}$ along the direction given by $\mathbf{e}_{1}$ :

$\mathbf{M}_{(i+1)}^{(0)}=\mathbf{M}_{(i)}+\Delta s . \mathbf{e}_{1}$

\subsubsection{Corrections}

The residual vector $\mathbf{R}$ at iteration $k$ is given by:

$$
\begin{gathered}
\underbrace{\mathbf{R}\left(\mathbf{Q}_{(i+1)}^{(k)}, \omega_{1(i+1)}^{(k)}, \omega_{2(i+1)}^{(k)}\right)}_{=\mathbf{R}^{(k)}}=\underbrace{\mathbf{Z}\left(\omega_{1(i+1)}^{(k)}, \omega_{2(i+1)}^{(k)}\right)}_{=\mathbf{Z}^{(k)}} \mathbf{Q}_{(i+1)}^{(k)} \\
+\underbrace{\mathbf{F}\left(\mathbf{Q}_{(i+1)}^{(k)}\right)}_{=\mathbf{F}^{(k)}}-\mathbf{P}
\end{gathered}
$$


As before, subscript $(i+1)$ will be dropped for clarity. Corrections are made orthogonally to the tangent vector $\mathbf{e}_{1}$ and the system at iteration $\mathrm{k}$ becomes:

$$
\left[\begin{array}{ccc}
\frac{\partial \mathbf{R}^{(k)}}{\partial \mathbf{Q}} & \frac{\partial \mathbf{R}^{(k)}}{\partial \omega_{1}} & \frac{\partial \mathbf{R}^{(k)}}{\partial \omega_{2}} \\
\Delta \hat{\mathbf{Q}}_{(i)}^{T} & \Delta \omega_{1(i)} & \Delta \omega_{2(i)}
\end{array}\right]\left[\begin{array}{c}
\Delta \mathbf{Q}^{(k+1)} \\
\Delta \omega_{1}^{(k+1)} \\
\Delta \omega_{2}^{(k+1)}
\end{array}\right]=\left[\begin{array}{c}
-\mathbf{R}^{(k)} \\
0
\end{array}\right]
$$

This system is underdetermined because of the presence of the additional unknown $\Delta \omega_{2}^{(k+1)}$. In fact, since $\theta_{2}=\omega_{2} t$ does not appear in the forcing term of the equations of motion (4), if $\mathbf{q}\left(\theta_{1}, \theta_{2}\right)$ is a solution then a phase-shifted function $\mathbf{q}\left(\theta_{1}, \theta_{2}+\sigma\right)$ is also a solution, for any value of $\sigma$, i.e. the phase is arbitrary. To define a unique solution an extra constraint is needed. This additional equation is often referred to as a "phase condition" and consists in fixing one of the variables other than $\omega_{1}$ and $\omega_{2}$, i.e. one component of vector $\mathbf{Q}$ or, within the Newton-Raphson procedure, one component of correction $\Delta \mathbf{Q}$. There is no systematic way for choosing the variable to be fixed. Several phase conditions can be found in textbooks dealing with periodic solutions [27, 28]. They can be considered as "orthogonality conditions" because they can be written as a null scalar product between the vector of unknowns and a vector $\mathbf{e}_{2}$ with non-null components along $\Delta \mathbf{Q}$ only, i.e. $\mathbf{e}_{2}=\left[\mathbf{e}_{2 Q}, 0,0\right]$. With this additional equation, the system to solve becomes:

$$
\left[\begin{array}{ccc}
\frac{\partial \mathbf{R}^{(k)}}{\partial \mathbf{Q}} & \frac{\partial \mathbf{R}^{(k)}}{\partial \omega_{1}} & \frac{\partial \mathbf{R}^{(k)}}{\partial \omega_{2}} \\
\Delta \hat{\mathbf{Q}}_{(i)}^{T} & \Delta \omega_{1(i)} \Delta \omega_{2(i)} \\
\mathbf{e}_{2 Q} & 0 & 0
\end{array}\right]\left[\begin{array}{c}
\Delta \mathbf{Q}^{(k+1)} \\
\Delta \omega_{1}^{(k+1)} \\
\Delta \omega_{2}^{(k+1)}
\end{array}\right]=\left[\begin{array}{c}
-\mathbf{R}^{(k)} \\
0 \\
0
\end{array}\right]
$$

Two constructions for $\mathbf{e}_{2}$ are proposed in the present paper, which differ in the way $\Delta \mathbf{Q}$ is constrained. The first choice is the simplest one and consists in directly fixing one arbitrarily chosen component of $\Delta \mathbf{Q}$ by considering the following phase equation:

$$
\frac{\partial \Delta q_{i}}{\partial \theta_{2}}(\boldsymbol{\theta})=0
$$

with $\Delta q_{i}$ being the displacement correction of the $i^{\text {th }}$ degree of freedom with $i$ arbitrarily chosen between 1 and $n$. This means that a zero phase shift relatively to the second hypertime dimension $\theta_{2}$ is forced for this particular degree of freedom. Once processed to the frequency domain, Eq. (21) becomes:

$$
\sum_{j=1}^{N} m_{j 2} \Delta Q_{2 j i}=0
$$

Using Eq. (22) is equivalent to using system (20) with the following $\mathbf{e}_{2}$ vector:

$$
\begin{gathered}
\mathbf{e}_{2}=[\underbrace{0, \ldots, 0}_{n}, \underbrace{0, \ldots, 0, m_{12}, 0, \ldots, 0}_{2 n}, \ldots, \underbrace{0, \ldots, 0, m_{j 2}, 0, \ldots, 0}_{2 n}, \\
\ldots, \underbrace{\left.0, \ldots, 0, m_{N 2}, 0, \ldots, 0,0,0\right]}_{2 n}
\end{gathered}
$$

with $m_{j 2}$ at $(n(2 j-1)+i)^{\text {th }}$ position, $j \in[1 . . N]$. With this phase equation, the system is well determined and the NewtonRaphson procedure can be carried out. This specific $\mathbf{e}_{2}$ vector is used for each iteration of the Newton-Raphson solver.

Alternatively, a second possible choice for $\mathbf{e}_{2}$ relies on geometric considerations. It consists in requiring the current correction to be orthogonal to the previous one as in the conjugate gradient procedure. Thus, vector $\mathbf{e}_{2}$ can be chosen as $\mathbf{e}_{2}^{(k)}=\left[\Delta \mathbf{Q}^{(k) T}, 0,0\right]$. For the applications considered in this paper, this approach appears slightly more effective than the previous one. However, the drawback here is that it is not possible to build vector $\mathbf{e}_{2}$ at the first iteration of the NewtonRaphson procedure. One way to overcome this problem is to perform a least square minimisation step on the under determined system (19) or to use the phase equation for the first iteration.

\subsection{Automatic harmonic selection}

Usually, simulations are carried out using an arbitrary set of harmonics. For instance, Chua and Ushida in [29] and Kim and Choi in [30] select harmonics as a function of an arbitrary number $M$. Harmonics are then chosen so that:

$\left|m_{j 1}\right|+\left|m_{j 2}\right| \leq M$

Legrand in [16] uses an even less restrictive formula:

$\left|m_{j 1}\right| \leq M$ and $\left|m_{j 2}\right| \leq M$

Using one of these formulae leads to use a large number of harmonics because the total number of harmonics rapidly increases with $M$ and the number of harmonics has a direct impact on performance. However many of these harmonics may be unnecessary and some important harmonics may not have been selected.

To overcome these problems, a technique for automatic selection of predominant harmonics can be implemented. Several selection criteria were proposed in the context of conventional periodic HBM. Among those, a simple selection procedure based on the error on the approximated nonlinear forces was presented by Laxalde in [31]. Another criterion was presented by Jaumouillé in [32] in order to take into account the global system behavior. It relies on the evolution of strain energy with an increasing number of harmonics. However, this incremental criterion requires an extra calculation cost for strain energy and the selection procedure can end prematurely if one harmonic has no contribution. An improved criterion based on local selection of harmonics per degree-of-freedom was proposed by Grolet [33] to overcome the limitations of previous procedures. However, this criterion requires a more complex numerical implementation. 


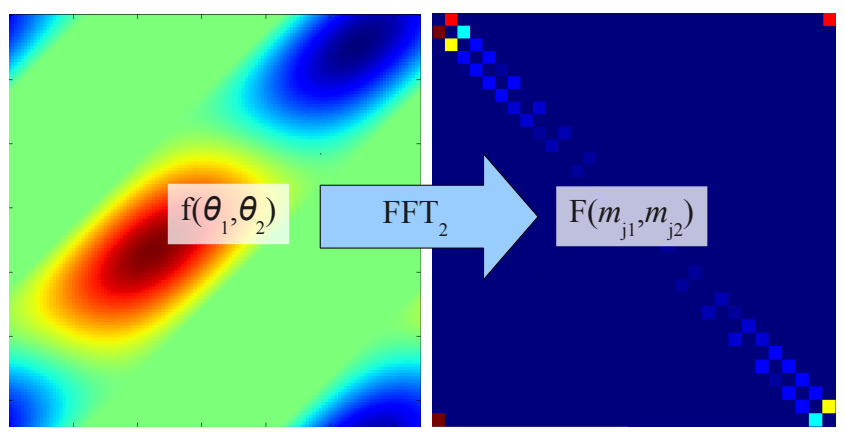

Fig. 14 Automatic harmonic selection, step 1.

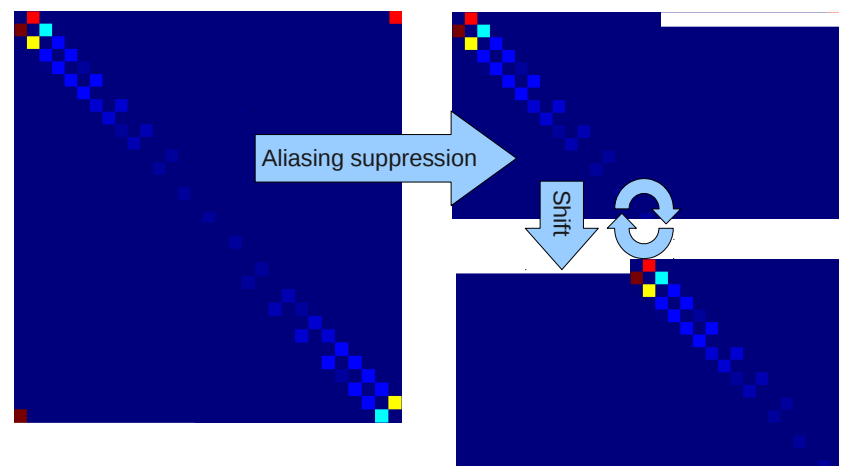

Fig. 15 Automatic harmonic selection, step 2.

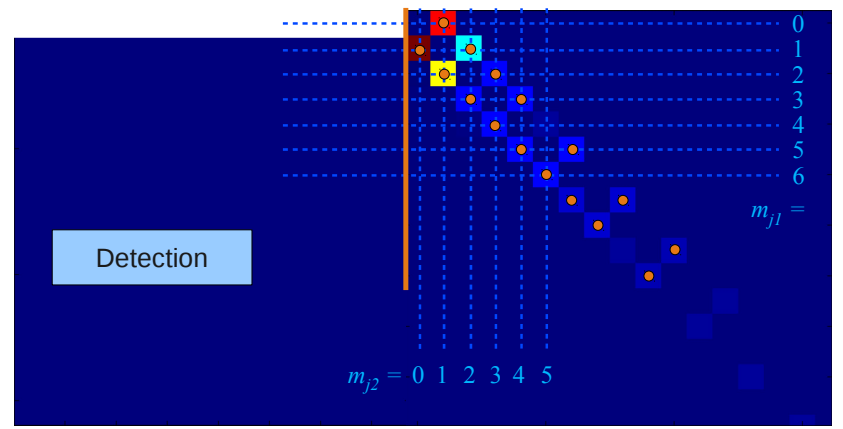

Fig. 16 Automatic harmonic selection, step 3.

The procedure described here applies to quasi-periodic bi-dimensional HBM. For the sake of simplicity of implementation, it also relies on the approximation of the nonlinear forces but differs from [31] in the way harmonics are selected. It consists in three steps.

Once a converged solution of the nonlinear system (17) is obtained for a given set of harmonics with the NewtonRaphson procedure described in section 4.2 :

- Step 1: Bi-dimensional FFT of nonlinear forces. The nonlinear forces are calculated in the time domain from displacements. In Fig. 14, the nonlinear forces are plotted as a function of $\theta_{1}$ and $\theta_{2}$ besides their FFT representation where each pixel represents a frequency component. A blue pixel represents a component with a very low contribution and a red pixel a high energy compo- nent. The spectrum yielded by FFT has aliasing and information is redundant.

- Step 2: Suppression of redundancy (see Fig. 15) and spectrum shift. The first half of rows or the first half of columns of the spectrum may be arbitrarily kept. For instance, here, the first half of rows are kept and the right half of the first row removed. For convenience, the right half of the spectrum is shifted with the left half.

- Step 3: Harmonics detection (see Fig. 16). The components with the highest contribution are extracted from the spectrum and added, one by one, until the error between nonlinear forces in the time domain and their reconstruction from the remaining harmonics equals a threshold value. The error is a norm of the difference between the two signals computed on the whole time vector. In other words, only important harmonics are retained. In the given example, sixteen harmonics are detected (indicated by an orange dot): $\{(0,1) ;(1,0) ;(1,2) ;(2,1) ;(2,3)$; $(3,2) ;(3,4) ; \ldots\}$. Despite being not nil, harmonic $(4,5)$ for instance is not retained due to a too small contribution.

The nonlinear system (17) is solved again using this new set of harmonics and the three steps are carried out again. This procedure is repeated until convergence of the harmonics. In general, one or two iterations are requiered for convergence. In the exemple presented here, this automatic selection procedure converged to a set of harmonics that verifies the empirical formulae (2) and (3). This proves the reliability of the procedure. With this procedure, only harmonics that really contribute to the response are used resulting to faster and more accurate simulations.

Both periodic and quasi-periodic HBM algorithms, with pseudo arc-length continuation and automatic harmonic selection, have been implemented in Code_Aster via Python user scripts which give access to Aster objects and commands like FEM elementary matrix computations.

\subsection{Application to the Jeffcott rotor}

Data from the time transient simulations, corresponding to one point of the branch, has been used to initialise the quasiperiodic HBM. Then the pseudo arc-length continuation procedure allows following the solution branch. Fig. 17 shows the same response curve than presented in Fig. 11 with the addition of the response curve given by the quasi-periodic HBM. The branch corresponding to the quasi-periodic partial rub phenomena is perfectly reproduced. The response curve given by the quasi-periodic HBM has been ploted in Fig. 18 as a function of $\Omega_{1}$ and $\Omega_{2}$. The evolution of $\Omega_{2}$ against $\Omega_{1}$ as yielded by the HBM has been ploted in Fig. 19. The evolution of $\Omega_{2}$ as a function of $\Omega_{1}$ found by the ex- 


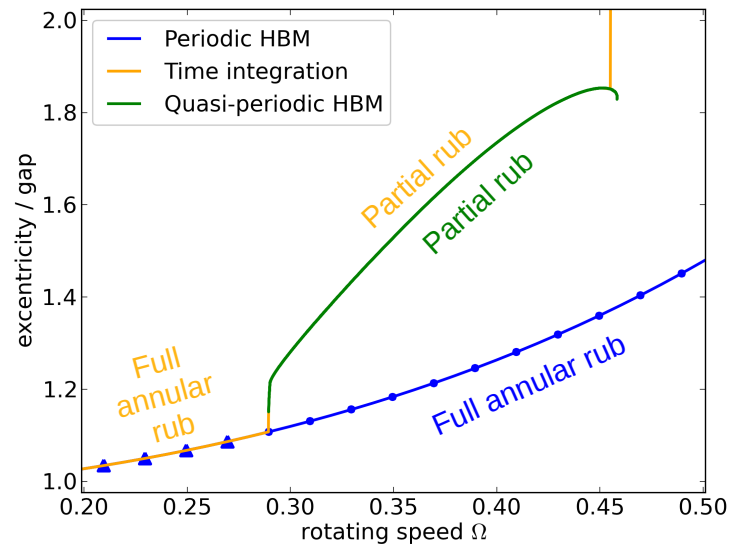

Fig. 17 Response curve including the quasi-periodic HBM response. Close up view. HBM stable (ム), HBM unstable (.).

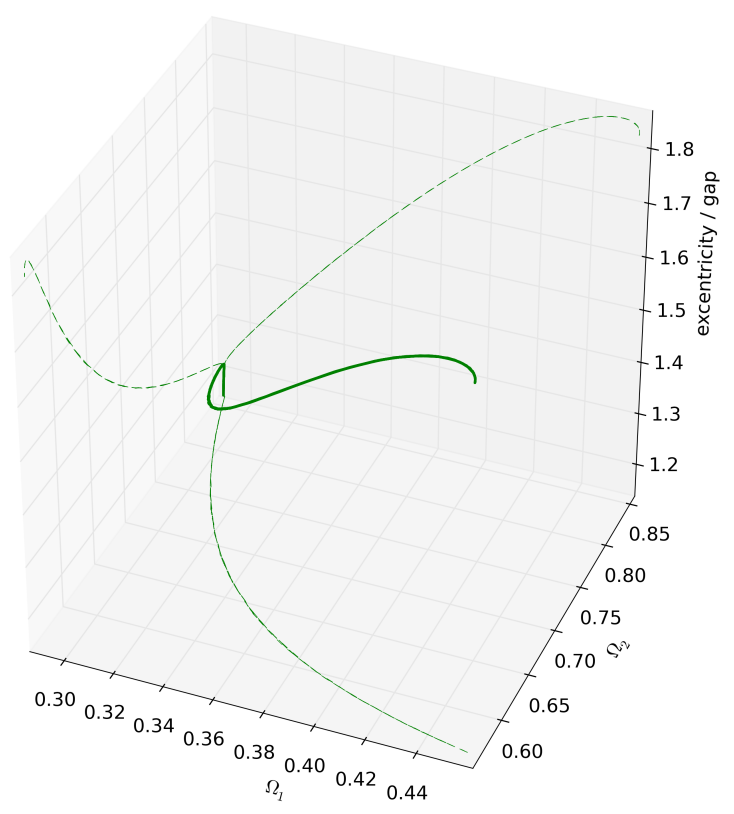

Fig. 18 Response curve given by quasi-periodic HBM as a function of $\Omega_{1}$ and $\Omega_{2}$. Projections of this curve onto the three plans are also ploted.

tended pseudo arc-length continuation method is very consistent with the evolution obtained from time integration.

Table 2 gives the computational time required to calculate the quasi-periodic partial rub branch using traditional time integration (Runge-Kutta) and the proposed quasi-periodic HBM. The proposed method appears to be more than seven times faster than the time integration method while providing very accurate results. It appeared that the AFT procedure is the most time consuming step. Bi-dimensional FFT are also more costly compared to the FFT used for periodic HBM.

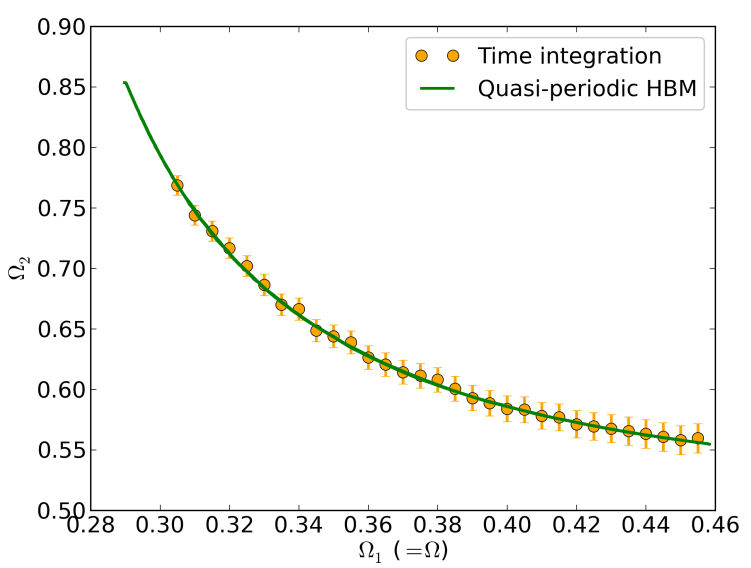

Fig. 19 Evolution of the second fundamental frequency $\Omega_{2}$ as a function of $\Omega_{1}$.

Table 2 Comparison of the CPU time required for the computation of the partial rub branch using the proposed method and traditional time integration.

\begin{tabular}{|c|c|c|}
\cline { 2 - 3 } \multicolumn{1}{c|}{} & $\begin{array}{c}\text { Time } \\
\text { integration }\end{array}$ & $\begin{array}{c}\text { Quasi-periodic } \\
\text { HBM }\end{array}$ \\
\hline Nb. of unknowns & 2 & 67 \\
\hline Nb. of points in branch & 36 & 274 \\
\hline CPU time (s) & 1336 & $184(7.30$ speed-up) \\
\hline
\end{tabular}

The backward whip branch should also be obtained from the quasi-periodic HBM technique as well and will be considered in the next developements.

\section{Conclusion}

The capabilities of the Harmonic Balance Method (HBM), with pseudo arc-length continuation and stability assessment, have been investigated for the computation of the steadystate behaviour of rotor-to-stator contact problems. The HBM results have been compared with classical time marching solutions and analytical results [11]. The application considers a simple Jeffcott rotor, with a circular, rigid, static stator. It has been demonstrated that although the rotor is submitted to unbalance only, there are four possible kinds of solution: two of these are periodic (no-rub motion and full annular rub), whereas the remaining two are quasi-periodic (partial rub and backward whip).

The periodic HBM is shown to be capable of accurately predict the two periodic solutions. The periodic HBM can also predict initiation of the quasi-periodic partial rub regime, which corresponds to a loss of stability of the periodic solution. However, the partial rub and backward whip motions cannot be described by means of the periodic HBM in the presented form.

An extension of the HBM to the treatment of quasi-periodic solutions has been proposed. This new method automati- 
cally determines the second fundamental frequency of the system which is the result of a self-excitation caused by contact. An extension of the pseudo arc-length continuation method made possible the continuation of a quasi-periodic branch despite the second frequency having an a priori unknown evolution along the branch. In addition, in order to improve both performance and accuracy of simulations, an automatic harmonic selection procedure has been proposed. With this procedure, only predominant harmonics are automatically used during simulation, requiring no intervention from the user. This method allowed to determine the partial rub branch and its results were very consistent with time integration results at a much lower cost.

The proposed quasi-periodic HBM currently requires to be initialised with data from time integration simulations. However, this requirement could be removed with the help of a branch switching procedure $[34,35]$ that would allow a smooth transition between periodic full annular rub and quasi-periodic partial rub. This possibility is currently being investigated.

Although results presented here concern mainly academic examples, the method has been successfully applied to industrial rotating machinery finite-element models [36].

Acknowledgements This work was partially supported by the French National Agency (ANR) in the framework of its Technological Research COSINUS program (IRINA, project ANR 09 COSI 00801 IRINA)

\section{References}

1. G. Jacquet-Richardet, M. Torkhani, P. Cartraud, F. Thouverez, T. Nouri Baranger, M. Herran, C. Gibert, S. Baguet, P. Almeida, L. Peletan, Rotor to stator contacts in turbomachines. review and application., Mech. Syst. Sig. Process. 40(2), 401-420 (2013)

2. S. Edwards, A.W. Lees, M.I. Friswell, The influence of torsion on rotor/stator contact in rotating machinery, J. Sound Vib. 225(4), $767-778$ (1999)

3. Z. Feng, X.Z. Zhang, Rubbing phenomena in rotor-stator contact, Chaos, Solitons Fractals 14(2), 257 - 267 (2002)

4. S. Popprath, H. Ecker, Nonlinear dynamics of a rotor contacting an elastically suspended stator, J. Sound Vib. 308(3-5), 767 - 784 (2007)

5. Q. Han, Z. Zhang, B. Wen, Periodic motions of a dual-disc rotor system with rub-impact at fixed limiter, Proc. Inst. Mech. Eng. C J. Mech. Eng. Sci. 222(10), 1935-1946 (2008)

6. X. An, J. Zhou, X. Xiang, C. Li, Z. Luo, Dynamic response of a rub-impact rotor system under axial thrust, Arch. Appl. Mech. 79(11), 1009-1018 (2009)

7. O. Grapis, V. Tamuzs, N.G. Ohlson, J. Andersons, Overcritical high-speed rotor systems, full annular rub and accident, J. Sound Vib. 290(3-5), 910 - 927 (2006)

8. S. Braut, R. Zigulic, A. Skoblar, G. Stimac, M. Butkovic, M. Jokic, Dynamic analysis of the rotor-stator contact due to blade loss, in 12th IFToMM World Congress. Besançon, France (2007)

9. W. Qin, Q. Chen, G. Meng, Nonlinear responses of a rub-impact overhung rotor, Chaos, Solitons Fractals 19(5), 1161 - 1172 (2004)
10. J. Jiang, H. Ulbrich, Stability analysis of sliding whirl in a nonlinear jeffcott rotor with cross-coupling stiffness coefficients, Nonlinear Dyn. 24(3), 269-283 (2001)

11. J. Jiang, Determination of the global responses characteristics of a piecewise smooth dynamical system with contact, Nonlinear Dyn. 57(3), 351-361 (2009)

12. D. Bently, P. Goldman, J. Yu, Full annular rub in mechanical seals, part ii: Analytical study, Int. J. Rotating Mach. 8(5), 329-336 (2002)

13. D. Childs, A. Bhattacharya, Prediction of dry-friction whirl and whip between a rotor and a stator, J. Vib. Acoust. 129(3), 355-362 (2007)

14. P. Sundararajan, S. Noah, An algorithm for response and stability of large order non-linear systems - application to rotor systems, J. Sound Vib. 214(4), 695 - 723 (1998)

15. S. Lau, Y. Cheung, S. Wu, Incremental harmonic balance method with multiple time scales for aperiodic vibration of nonlinear systems, J. Appl. Mech. 50(4), 871-876 (1983)

16. M. Legrand, Modèles de prédiction de l'intéraction rotor/stator dans un moteur d'avion. Ph.D. thesis, École Centrale de Nantes (2005)

17. M. Legrand, S. Roques, C. Pierre, B. Peseux, P. Cartraud, ndimensional harmonic balance method extended to non-explicit nonlinearities, Rev. Eur. Mec. Num. 15, 269-280 (2006)

18. N. Coudeyras, S. Nacivet, J.J. Sinou, Periodic and quasi-periodic solutions for multi-instabilities involved in brake squeal, J. Sound Vib. 328(4-5), 520 - 540 (2009)

19. D. Laxalde, F. Thouverez, Complex non-linear modal analysis for mechanical systems: Application to turbomachinery bladings with friction interfaces, J. Sound Vib. 322(4-5), 1009 - 1025 (2009)

20. M. Guskov, J.J. Sinou, F. Thouverez, Multi-dimensional harmonic balance applied to rotor dynamics, Mech. Res. Commun. 35(8), $537-545$ (2008)

21. F. Schilder, W. Vogt, S. Schreiber, H. Osinga, Fourier methods for quasi-periodic oscillations, Int. J. Numer. Meth. Engng. 67(5), 629-671 (2006)

22. EDF R\&D, Code_aster: A general code for structural dynamics simulation under gnu gpl licence. http://www.code-aster.org (2001-)

23. E. Jones, T. Oliphant, P. Peterson, et al., Scipy: Open source scientific tools for Python. http://www.scipy.org/ (2001-)

24. E. Petrov, D. Ewins, Analytical formulation of friction interface elements for analysis of nonlinear multi-harmonic vibrations of bladed disks, J. Turbomach. 125(2), 364-371 (2003)

25. T. Cameron, J. Griffin, An alternating frequency/time domain method for calculating the steady-state response of nonlinear dynamic systems, J. Appl. Mech. 56(1), 149-154 (1989)

26. L. Peletan, S. Baguet, M. Torkhani, G. Jacquet-Richardet, A comparison of stability computational methods for periodic solution of nonlinear problems with application to rotordynamics, Nonlinear Dyn. 72(3), 671-682 (2013)

27. A. Nayfeh, B. Balachandran, Applied Nonlinear Dynamics: Analytical, Computational, and Experimental Methods. Wiley, New York (1995)

28. E. Doedel, Lecture notes on numerical analysis of nonlinear equations, in Numerical Continuation Methods for Dynamical Systems, ed. by B. Krauskopf, H. Osinga, J. Galan-Vioque, Springer Netherlands, pp. 1-49 (2007)

29. L. Chua, A. Ushida, Algorithms for computing almost periodic steady state response of non-linear systems to multiple input frequencies, IEEE Trans. Circuits Syst. 28, 953-971 (1981)

30. Y.B. Kim, S.K. Choi, A multiple harmonic balance method for the internal resonant vibration of a non-linear jeffcott rotor, J. Sound Vib. 208(5), 745 - 761 (1997)

31. D. Laxalde, Etude d'amortisseurs non-linéaires appliqués aux roues aubagées et aux systèmes multi-étages. Ph.D. thesis, École Centrale Lyon (2007) 
32. S. Jaumouille, J. Sinou, B. Petitjean, An adaptive harmonic balance method for predicting the nonlinear dynamic responses of mechanical systemsapplication to bolted structures, J. Sound Vib. 329, 4048-4067 (2010)

33. A. Grolet, F. Thouverez, On a new harmonic selection technique for harmonic balance method, Mech. Syst. Sig. Process. 30(0), 43$60(2012)$

34. W. Beyn, A. Champneys, E. Doedel, A. Kuznetsov Y.U., W. Govaerts, B. Sandstede, Numerical continuation, and computation of normal forms, Elsevier, Handbook of Dynamical Systems, vol. 2, chap. 4, pp. 149-219 (2002)

35. R. Seydel, Practical Bifurcation and Stability Analysis. Interdisciplinary Applied Mathematics. Springer (2010)

36. L. Peletan, Stratégie de modélisation simplifiée et de résolution accélérée en dynamique non linéaire des machines tournantes. application au contact rotor-stator. Ph.D. thesis, INSA Lyon, 2012ISAL-0146 (2012) 